We agree that depression following myocardial infarction predicts long-term quality of life and we recently showed that this effect persists after controlling for cardiac condition and quality of life at 3 months post-myocardial infarction (de Jonge et al, 2006). However, it is unclear whether and how detection and treatment of depression can counter these effects. In the SADHART study Glassman et al (2002) found that the effects of sertraline were modest and appeared to be restricted to depression with an onset before the infarction, but Dickens et al found that depression and anxiety which were present before myocardial infarction did not predict quality of life. In the ENRICHD trial (Berkman et al, 2003), cognitivebehavioural therapy had modest effects on depressive symptoms at 6 months postinfarction in patients with depression and social isolation, but these effects diminished over time. In the EXIT trial (Appels et al, 2005), where the focus of treatment was explicitly on vital exhaustion, only some intervention effects were observed and these were modified by the presence of a previous cardiac history.

We agree with Dickens et al that there is a need for improved detection and treatment of depression and anxiety following myocardial infarction but several questions need to be addressed. These include 'can the effects of depression and anxiety be linked to specific subgroups of emotional disorders based on symptoms and/or onset?; 'can interventions that were developed in general psychiatry be applied to depression post-myocardial infarction or should they be adapted?'; and 'how can psychiatric interventions be integrated into regular cardiac aftercare?'

Appels, A., Bar, F., van der Pol, G., et al (2005)

Effects of treating exhaustion in angioplasty patients on new coronary events: results of the randomized Exhaustion Intervention Trial (EXIT). Psychosomatic Medicine, 67, 217-223.

Berkman, I. F., Blumenthal, I., Burg, M., et al (2003) Effects of treating depression and low-perceived social support on clinical events after myocardial infarction the enhancing recovery in coronary heart disease patients (ENRICHD) randomized trial. JAMA, 289 3106-3116.

de Jonge, P., Spijkerman, T. A., van den Brink, R. H. S., et al (2006) Depression following myocardial infarction is a risk factor for declined health-related quality of life and increased disability and cardiac complaints at 12 months. Heart, 92, 32-39.

Dickens, C. M., McGowan, L., Percival, C., et al (2006) Contribution of depression and anxiety to impaired health-related quality of life following first myocardial infarction. British Journal of Psychiatry, 189, 367-372.

Glassman, A. H., O'Connor, C. M., Califf, R. M., et al (2002) Sertraline treatment of major depression in patients with acute $\mathrm{MI}$ or unstable angina. JAMA, $\mathbf{2 8 8}$ 70I-709.

P. de Jonge Department of Internal Medicine and Department of Psychiatry, University Medical Centre Groningen, University of Groningen, Hanzeplein I, P.O. Box 30.00I, Groningen, The Netherlands. Email: Peter.de.Jonge@med.umcg.nl

J. Ormel Department of Psychiatry, University Medical Centre Groningen, The Netherlands doi: 10.II92/bjp.190.3.272b

Authors' reply: We agree that although observational studies have shown that depression is associated with subsequent impairment in health-related quality of life in coronary heart disease, intervention studies have failed to provide convincing proof that treating depression improves this outcome. Previous intervention studies have not addressed this question satisfactorily because the SADHART study (Glassman et al, 2002) was not sufficiently powered to demonstrate the efficacy of antidepressants in coronary heart disease and the ENRICHD study (Berkman et al, 2003) did not anticipate very high rates of spontaneous remission of depression or unplanned prescription of antidepressants in the control group. The results of these trials, however, together with our own results are valuable for planning future treatment trials.

We also agree that there are many unanswered questions relating to the nature of the association between depression and negative outcomes in coronary disease. As mentioned by de Jonge \& Ormel, the timing of the onset of depression (Dickens et $a l, 2004 a$ ), the specific aspects of depression or anxiety that are associated with poor outcome and the possibility of vulnerable sub-populations of patients (such as those without social support) (Dickens $e t$ $a l, 2004 b)$ require further investigation. Furthermore, whether the association between depression and negative outcomes in coronary disease is the result of residual confounding by severity of heart disease (Dickens et al, 2005) remains unsolved. Further research is required to address these questions, although it is likely that most will only be convincingly resolved through intervention studies.
Dickens, C. M., Percival, C., McGowan, L., et al

(2004a) The risk factors for depression in first myocardial infarction patients. Psychological Medicine, 34, $1083-1092$.

Dickens, C. M., McGowan, L., Percival, C., et al (2004b) Lack of a close confidant, but not depression, predicts further cardiac events after myocardial infarction. Heart, 90, 518-522.

Dickens, C., McGowan, L., Percival, C., et al (2005) Association between depressive episode before first myocardial infarction and worse cardiac failure following infarction. Psychosomatics, 46, 523-528.

C. Dickens Department of Psychiatry, Rawnsley Building, Manchester Royal Infirmary, Oxford Road, Manchester MI3 9WL, UK. Email: chris.dickens@manchester.ac.uk

F. Creed Department of Psychiatry, Manchester Royal Infirmary, Manchester, UK

doi: 10.I192/bjp.190.3.273

\section{High female suicide rates: ecological fallacy or sad reality?}

Yip \& Liu (2006) present a demographic perspective of female suicide in China, the only country in which the suicide rate is higher among women than men. However, this reversed gender representation also exists in certain communities in other countries. In the Indian subcontinent suicide rates are higher in men than in women but the difference is lower than in most countries: the male:female suicide ratio in India is 1.3:1 (Cheng \& Lee, 2000). Suicide among immigrants from the Indian subcontinent to Britain was higher among young married women than men (Soni Raleigh et al, 1990). Tadros \& Salib (2006) also reported that significantly more Asian women than Asian men killed themselves in Birmingham and Solihull, a clearly reversed gender ratio compared with suicide in the White population and in other ethnic groups in Birmingham and the UK as a whole.

Suicide terrorism is not an egoistic suicide but none the less is a form of fatal self-harm in the legal and human sense and has a distinct underlying political, individual and social logic. The support of and acceptance by the attackers' own communities ensure an endless supply of volunteers who seek 'voluntary violent death' in a bizarre act of so-called martyrdom, in order to promote what they firmly believe to be a just cause. Women carried out $15 \%$ (64) of such attacks over the past 25 years (Pope, 2005). Chechen women carried out $60 \%$ of all suicide bombings in Russia 
and $70 \%$ of such attacks were executed by Kurdish women in Turkey (Pope, 2005). There is also a high proportion of women suicide bombers in the Tamil Tigers $(30 \%)$. al Qa'ida, which associates itself with Islamic fundamentalism, never used female suicide attackers from its formation in 1993 until the tragic attack in Jordan in 2005.

In general, women are at a lower risk of suicide than men and a protective effect of child-bearing in terms of suicide risk has been postulated (Catalan, 2000). This does not appear to apply to female suicide bombers or to some countries and cultures in which gender representation in suicide is reversed. A higher female:male suicide ratio is not unique to China. The significantly higher rate of female suicide observed outside China is not an 'ecological fallacy' but a sad reality.

Catalan, J. (2000) Sexuality, reproductive cycle and suicidal behaviour. In International Handbook of Suicide and Attempted Suicide (eds K. Hawton \& K. van Heeringen), pp. 294-307. John Wiley \& Sons.

Cheng, A. T. A. \& Lee, C. (2000) Suicide in Asia and the Far East. In International Handbook of Suicide and Attempted Suicide (eds K. Hawton \& K. van Heeringen), pp. 29-48. John Wiley \& Sons.

Pope, R. A. (2005) Dying to Win. The Strategic Logic of Suicide Terrorism, pp. 203-216. Random House.

Soni Raleigh, V., Bulusu, L. \& Balarajan, R. (1990)

Suicides among immigrants from the Indian

subcontinent. British Journal of Psychiatry, 156, 46-50.

Tadros, G. \& Salib, E. (2006) Elderly suicide in primary care. International Journal of Geriatric Psychiatry, doi: 10.1002/gps.1734 (Epub ahead of print)

Yip, P. S. F. \& Liu, K. Y. (2006) The ecological fallacy and the gender ratio of suicide in China. British Journal of Psychiatry, 189, 465-466.

E. Salib Peasley Cross Hospital, St Helens, and Honorary Senior Lecturer, Liverpool University Email: esalib@hotmail.com

G. Tadros Birmingham and Solihull Mental Health Trust, Birmingham, UK

doi: 10.I192/bjp.190.3.273a

Authors' reply Salib \& Tadros highlight the important issue of high female suicide rates among Indian migrants and the use of female suicide bombers. Like the high suicide rates among young females in rural China (Yip \& Liu, 2006), these deviations from the general pattern should not be discounted as mere exceptions but should be considered as representative of the distressing situations faced by some women in Asia.
We also believe that socio-economic deprivation and poor social support - the 'sad reality' - faced by young women in rural China are underlying causes of the high suicide rates. Like the young married Indian women in Britain, there is some indication that young married women in rural China might be at high risk (Pearson et al, 2002). This reminds us that the lives of married women differ greatly across regions, countries, cultures and economies, and there is a need to avoid oversimplification when describing suicide in different countries; one size does not fit all.

Over $60 \%$ of the world's suicides occur in Asian countries where low male:female ratios for suicide are common (Yip et al, 2000). Although the official male:female ratio for suicide in India was still greater than 1 (1.2:1 in 2002), the ratio was 0.8 among those aged 14 or below (World Health Organization, 2006). However, unlike China (Yip \& Liu, 2006), the small size of this population subgroup meant that the national male:female ratio remained greater than 1. (This is the essence of our ecological fallacy argument.) In addition to specific social factors, the similarity in the methods of suicide used by males and females, together with the poor access to medical facilities, might explain the low male:female ratio in India and China. Restricting access to pesticides will prevent many suicides in Asia. In the long term improving economic and educational opportunities, especially for rural women in deprived areas, raising awareness of depression and better treatment will be pivotal for preventing suicides.

Pearson, V., Phillips, M. R., He, F., et al (2002) Attempted suicide among young rural women in the People's Republic of China: possibilities for prevention Suicide and Life Threatening Behavior, 32, 359-369.

Yip, P. S., Callanan, C. \& Yuen, H. P. (2000) Urban/ rural and gender differentials in suicide rates: east and west. Journal of Affective Disorders, 57, 99-106.

World Health Organization (2006) Country Report on Suicide (India).World Health Organization. http: / / www.who.int/entity/mental_health/media/indi.pdf

K. Y. Liu Nuffield College, University of Oxford, Oxford, UK

P. S. F. Yip Hong Kong Jockey Club Centre for Suicide Research and Prevention, University of Hong Kong, Hong Kong. Email: sfpyip@hku.hk doi: 10.1192/bjp.190.3.274

\section{Self-poisoning with pesticides in India}

Bertolote et al (2006) report the global response to deaths from pesticide poisoning. Suicide rates in southern India have been reported to be high (Joseph et al, 2003; Aaron et al, 2004; Abraham et al, 2005; Prasad et al, 2006), with 1741 suicides documented in a population of about 100000 from 1986 to 2005. Hanging (804 of $1741,46.2 \%)$ and poisoning (746 of $1741,42.8 \%$ ) were the methods most commonly employed. Although people under 40 years tended to use poisons, older people tended to choose hanging $\left(\chi^{2}=36.71\right.$, d.f. $\left.=4, P<0.001\right)$. Significantly more males (465 of $984,47.3 \%$ ) than females (281 of $757,37.1 \%) \quad\left(\chi^{2}=17.6\right.$, d.f. $=1, P<0.001)$ chose death by poisoning. There was no significant change in the overall rate of suicide or the method employed during the period. Detailed analysis of the data from 2001 to 2005 revealed that only $68 \%$ of the fatal episodes of self-poisoning were a result of ingestion of pesticides.

Self-poisoning with pesticides is a significant public health problem in low- and middle-income countries. The majority of such poisoning occurs in rural agrarian households. Some suggestions to reduce such deaths are currently difficult to implement. Enforcing the hazardous chemicals and wastes conventions to restrict and control the sale and use of pesticides in such regions is no small task and requires major political, administrative, financial and social commitment. Given the many competing demands on limited governmental resources in low- and middle-income countries, such protocols are difficult to implement. In addition, the improved recognition and treatment of mental illness may not have a significant impact on the overall suicide rate as many people in the low- and middle-income countries who die by suicide do not have severe mental illness. Rather, the majority of such attempts are impulsive and follow stressful life events. Although reducing accessibility to pesticides will decrease such impulsive attempts and consequent deaths, social, economic and cultural factors must also be addressed to make a real difference. Thus, although the World Health Organization's intersectoral global initiative is a step in the right direction, it is imperative that practical issues related to its implementation are discussed. It is necessary to consider 